\title{
Design and Construction of a Realistic DWI Phantom for Filtering Performance Assessment
}

\author{
Antonio Tristán-Vega and Santiago Aja-Fernández \\ Laboratory of Image Processing, University of Valladolid. Spain \\ atriveg@lpi.tel.uva.es, sanaja@tel.uva.es
}

\begin{abstract}
A methodology to build a realistic phantom for the assessment of filtering performance in Diffusion Weighted Images (DWI) is presented. From a real DWI data-set, a regularization process is carried out taking into account the diffusion model. This process drives to a model which accurately preserves the structural characteristics of actual DWI volumes, being in addition regular enough to be considered as a noise-free data-set and therefore to be used as a ground-truth. We compare our phantom with a kind of simplified phantoms commonly used in the literature (those based on homogeneous cross sections), concluding that the latter may introduce important biases in common quality measures used in the filtering performance assessment, and even drive to erroneous conclusions in the comparison of different filtering techniques.
\end{abstract}

\section{Introduction}

Diffusion Tensor Imaging (DTI) allows the study of the fiber tracts in the white matter of the brain in vivo. DTI are obtained from Diffusion Weighted Images (DWI); each of them is acquired by applying a sensitizing gradient in a known direction, which produces an attenuation in the T2 (baseline) image following an exponential law [1. By collecting six or more independent gradient directions, the underlying fiber structure at each voxel may be inferred in terms of the six free components of the Diffusion Tensor (DT) [2]. As opposed to conventional MRI, DWI show a poor Signal to Noise Ratio (SNR), since the signal power is lower due to the DWI attenuation. This is especially the case with modern High Angular Resolution Diffusion Imaging (HARDI) techniques, where very strong gradients (and therefore very strong attenuation) have to be applied in order to improve the angular contrast [3. Moreover, the Rician nature of the noise in DWI [4] prevents the use of conventional Gaussian-based filtering techniques.

The adverse effect of Rician noise in DWI has been previously reported in [56], where it has been shown that it produces artifacts which hinder the recovery of the original signal once the DT has been estimated. To palliate the effect of noise, a number of techniques may be used, including regularization after DT estimation 7, regularized estimation of the DT 8] and DWI restoration before DT estimation. In this last category, a number of techniques have been proposed: the Conventional Approach [9] (CA), based on the properties of the second order moment of Rician data; Maximum Likelihood (ML) estimation 10; anisotropic diffusion 11]; wavelets 12]; total variation [13]; Unbiased Non Local Means (UNLM) 
[14 15]; Linear Minimum Mean Squared Error (LMMSE) filtering [16]17]; multichannel, Rician-corrected Wiener filtering [18, and others.

The quantitative assessment of filtering performance has been carried out in different ways. They include visual assessment [14151617] and indirect measures based on the properties of the DTI volumes recovered from filtered DWI 1618; in this case, the computation of related parameters such as the smoothness in the fiber tracts estimated from DTI 18 make it difficult to evaluate the filtering performance, since the final result depends on a number of factors others than the filtering itself. On the other hand, direct evaluation on the filtered DWI is a difficult task; in [14 12 acquisitions of the same patient are available, so a leave-one-out strategy is used to filter one of the volumes each time and compare the result to a noise-free image obtained from the remaining 11 volumes. When multiple observations are not available, synthetic data has to be used. As opposed to conventional MRI, for which very realistic phantoms exist and have been intensively used 19, there is not a DWI synthetic data-set widely accepted as a standard, so over-simplified models are used like in [151617], based on large homogeneous regions simulating coarse crossing fiber tracts. Commonly used configurations are crossing sections in two [151618] and three [17] dimensions, although other approaches like the "Earth" and "logarithm" in 18 or the logmarithmic spiral in [16] are possible. Nevertheless, these simple configurations are not representative of the complex architecture of the white matter of the brain, which in general presents multiple fiber crossings, bending and sharp changes in curvature/orientation in the space of a few voxels. Thus, we propose a novel methodology to design a highly realistic DWI phantom to be used as a gold standard for filtering performance assessment. It is built from a real DWI data-set, so it is able to account for the high complexity of the human brain, but at the same time we use a number of regularization techniques which allow us to obtain a nearly noise-free signal which may be used as a ground truth. Additionally, we empirically show that commonly used phantoms may bias the results on performance assessment, driving to erroneous conclusions. We illustrate this by the comparison of two popular DWI filtering schemes (UNLM and LMMSE) based on a conventional phantom and the one here introduced.

\section{Materials}

The phantom has been built from a real DWI volume of a volunteer. We use a SENSE EPI data-set scanned in a 3 Tesla General Electrics Echospeed system. (Sequence: Maximum gradient amplitudes: $40 \mathrm{mT} / \mathrm{M}$. Rectangular Field of view of 220 x $165 \mathrm{~mm}$. Slice thickness $1.7 \mathrm{~mm}$. Receiver bandwidth: $6 \mathrm{kHz}$. TE $70 \mathrm{~ms}$; TR $80 \mathrm{~ms}$ (effective TR $2500 \mathrm{~ms}$ )). It comprises 8 non-weighted baseline images and 51 gradient directions, and is $256 \times 256 \times 81$ voxels in size, with a resolution of $0.94 \times 0.94 \times 1.7 \mathrm{~mm}^{3}$. The $b$ value is $586 \mathrm{~s} / \mathrm{mm}^{2}$; although this value is quite small for practical applications (typical values are greater than $1000 \mathrm{~s} / \mathrm{mm}^{2}$ ), it has the advantage that its SNR is also greater. 


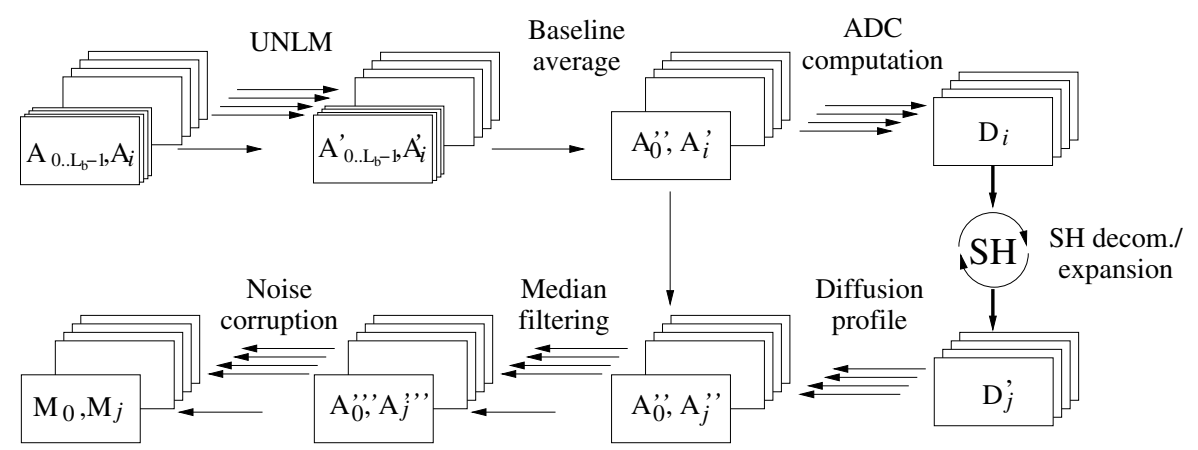

Fig. 1. Overview of the construction of the synthetic phantom. The input is the original (real) DWI volume, and the output the synthetic volume.

\section{Method}

\subsection{Overview}

Fig. 1 summarizes the steps on the design of the phantom, which we detail later on. We filter the 8 baseline images $\left(A_{0} \ldots A_{L_{b}-1}\right.$, with $\left.L_{b}=8\right)$ and the 51 gradient directions $\left(A_{L_{b}} \ldots A_{L_{b}+L_{g}-1}\right.$, with $\left.L_{g}=51\right)$ to reduce the amount of noise $\left(A_{0}^{\prime} \ldots A_{L_{b}+L_{g}-1}^{\prime}\right)$. The 8 baselines are then averaged to obtain one single, nearly noise-free baseline $\left(A_{0}^{\prime \prime}\right)$. All gradient directions are divided by the baseline to obtain the attenuation signal, and from it the Apparent Diffusion Coefficient (ADC) by taking its logarithm $\left(D_{1} \ldots D_{L_{g}}\right)$. We use a Spherical Harmonics representation to regularize the $\mathrm{ADC}$. This smoothed $\mathrm{ADC}\left(D_{1}^{\prime} \ldots D_{L_{g}^{\prime}}^{\prime}\right)$, together with the noise-free baseline $A_{0}^{\prime \prime}$ is used to produce synthetic gradient directions $\left(A_{1}^{\prime \prime} \ldots A_{L_{g}^{\prime}}^{\prime \prime}\right)$. The resulting DWI volume is median filtered $\left(A_{0}^{\prime \prime \prime} \ldots A_{L_{g}^{\prime \prime}}^{\prime \prime \prime}\right)$ to remove outliers and obtain the noise-free ground truth. The input to the filters will be the noise-corrupted phantom $\left(M_{0} \ldots M_{L_{g}^{\prime}}\right)$.

\subsection{Filtering and Baseline Average}

Although the low $b$ value of the DWI volume produces a relatively high SNR, a previous filtering is needed to drastically reduce noise. We use the popular UNLM as described in [15, with a search radius of 5 voxels, a comparison radius of 2 voxels, and $h=\sigma$. Assuming that the residual noise in the baselines after filtering is of zero mean, we average $A_{0}^{\prime} \ldots A_{L_{b}-1}^{\prime}$ to obtain $A_{0}^{\prime \prime}$, which is assumed to be noise-free. The gradient images have lower SNR, so they need further regularization taking into account the diffusion model, as explained next.

\subsection{Computation of the Apparent Diffusion Coefficient}

Under the assumption of Gaussian diffusion, the (true) diffusion signal $E_{i}$ is related to the (true) baseline $E_{0}$ by the Stejskal-Tanner equation [1]:

$$
E_{i}=E_{0} \exp \left(-b \cdot g_{i}^{T} \mathcal{D} g_{i}\right), i=1 \ldots L_{g}
$$


with $g_{i}$ the $i$-th direction and $\mathcal{D}$ the diffusion tensor. However, this equation cannot model the diffusion process in the event of complex configurations such as fiber crossing or bending 3 . In this case, the diffusion may be represented in terms of a positive function $D$ defined on the unit sphere, the ADC:

$$
E_{i}=E_{0} \exp \left(-b \cdot D\left(g_{i}\right)\right) \Rightarrow D\left(g_{i}\right) \simeq-\log \left(A_{i}^{\prime} / A_{0}^{\prime \prime}\right) / b, i=1 \ldots L_{g}
$$

\subsection{Analysis in the Basis of Spherical Harmonics}

Since the ADC is defined on the unit sphere, it may be represented in the basis of Spherical Harmonics. We use the regularized least squares approach in 3 with $\lambda=0.006$ and order 6 (28 basis functions), which in our experiments showed the best trade-off between representation capability (i.e. it does not hinder the ability to resolve crossings or alter the angular contrast) and regularization. This way, the noise-free (due to least squares regularization) $\mathrm{ADC}$ is:

$$
D^{\prime}(g)=\sum_{l=0}^{27} C_{l} Y_{l}(g)
$$

where $Y_{l}$ are the basis functions and $C_{l}$ are the coefficients of the expansion obtained from $D\left(g_{i}\right)$ in eq. (2) (see 3] for details). Note that the basis functions $Y(g)$ are continuous-valued. Therefore, once we have chosen an arbitrary set of gradient directions $g_{j}^{\prime}, j=1 \ldots L_{g}^{\prime}$, the noise free diffusion signal is, see eq. (2):

$$
A_{j}^{\prime \prime}=A_{0}^{\prime \prime} \exp \left(-b^{\prime} \sum_{l=0}^{27} C_{l} Y_{l}\left(g_{j}^{\prime}\right)\right), j=1 \ldots L_{g}^{\prime}
$$

The set of gradient directions $g_{j}^{\prime}$ and the magnitude of the sensitizing gradients $b^{\prime}$ are not necessarily the same as the original ones, $g_{i}$ and $b$. In fact, we use a more realistic value for practical applications, $b^{\prime}=1200 \mathrm{~s} / \mathrm{mm}^{2}$. For $g_{j}$, we generate 15 gradient directions as antipodal pairs.

\subsection{Median Filtering}

As a final processing, all gradient images and the baseline are filtered slice-byslice with a median filter of size $3 \times 3$. This step is required to remove isolated outliers due to a poor fit of the spherical harmonics. Although very few voxels are affected by this artifact, this is useful to achieve a visually adequate result.

\subsection{Noise Corruption}

To produce the inputs to the filter, we corrupt the image with Rician noise 4]:

$$
M_{j}=\sqrt{\left(A_{j}^{\prime \prime \prime}+n_{c}\right)^{2}+n_{s}^{2}}, j=0 \ldots L_{g}^{\prime}
$$

where $n_{c}$ and $n_{s}$ are independent Gaussian random processes with zero mean and power $\sigma^{2}$. The noise in MRI is usually not white, but it is correlated inside each slice due to the spatial interpolation produced by the zero padding of the 

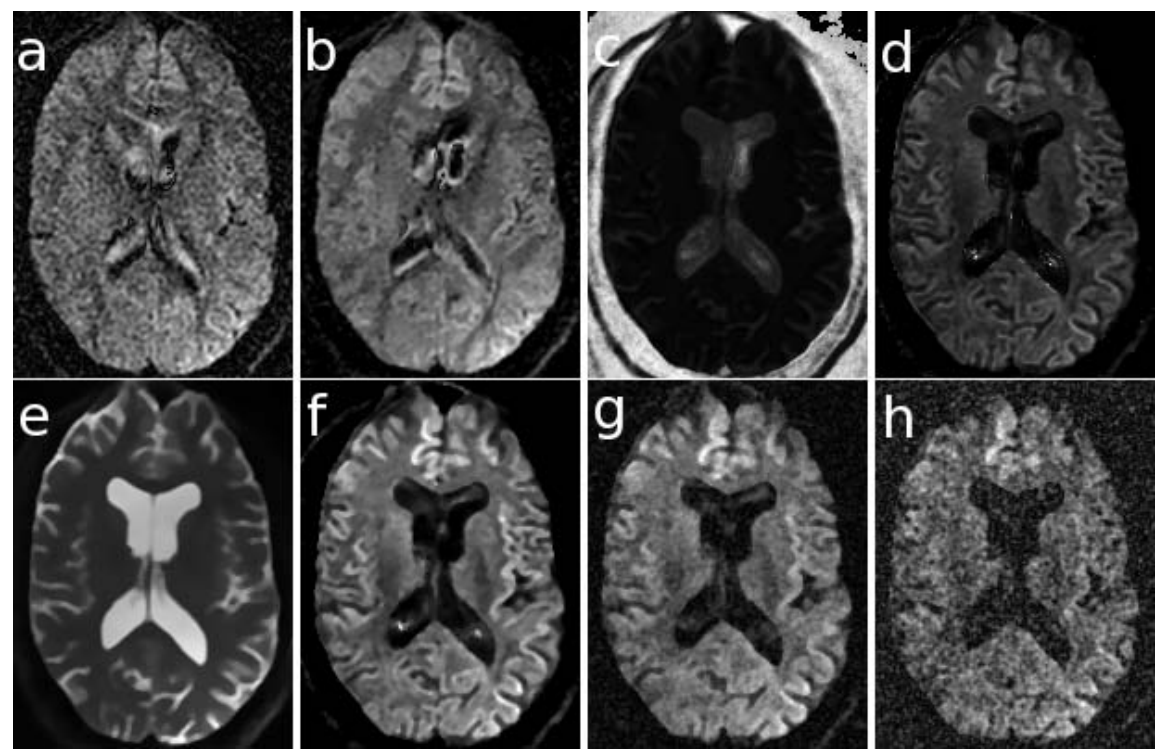

Fig. 2. Illustration of the whole process of phantom construction; from the original gradients $A_{i}(\mathbf{a})$, UNLM is used to drastically reduce noise $\left(A_{i}^{\prime}\right.$ in $\left.\mathbf{b}\right)$. Then the ADC $\left(D_{i}\right)$ is computed and regularized $\left(D_{j}^{\prime}\right)$ via spherical harmonics decomposition, with a total of 28 coefficients $C_{l}$ (c shows the first one). Arbitrary gradient directions $A_{j}^{\prime \prime}$ are generated with a higher $b$ value $(\mathbf{d})$. Finally, a median filter is used to obtain the noise-free phantom $A_{j}^{\prime \prime \prime}$, comprising the averaged baseline $A_{0}^{\prime \prime \prime}(\mathbf{e})$ and each of the 15 gradient images $A_{j}^{\prime \prime \prime}(\mathbf{f})$. Correlated noise is added to produce the noisy phantoms $M_{i}$, with a maximum SNR of $54 \mathrm{~dB}(\mathbf{g})$ and a minimum of $48 \mathrm{~dB}(\mathbf{h})$ (Original: $51.7 \mathrm{~dB}$ ).

spectrum of the image in the $k$-space. To account for this characteristic, we generate white noise processes $\tilde{n}_{c, s}$ and low-pass filter them inside each slice with a $2-\mathrm{D}$ Gaussian kernel $G\left(p_{1}, p_{2}\right)$ in the spatial (image) domain. It has an isotropic variance $\eta^{2}$ :

$$
n_{c, s}\left(p_{1}, p_{2}\right)=\left(\sum_{q_{1}, q_{2}} G^{2}\left(q_{1}, q_{2}\right)\right)^{-1} \sum_{q_{1}, q_{2}} \tilde{n}_{c, s}\left(q_{1}, q_{2}\right) G\left(p_{1}-q_{1}, p_{2}-q_{2}\right)
$$

where dividing by the energy of $G$ is necessary to keep the noise power constant.

\section{Results and Discussion}

We show in Fig. 2 an illustration of the whole process described in Section 3 . First, note that although UNLM is able to achieve very clean images (compare $\mathbf{a}$ and $\mathbf{b}$ ), the filtered image is not an adequate phantom: there is some residual noise, and the contrast of the image is very poor. This justifies the need for the regularization of the $\mathrm{ADC}(\mathbf{c}$ and $\mathbf{d})$ and the increase in $b$. As a result, the 


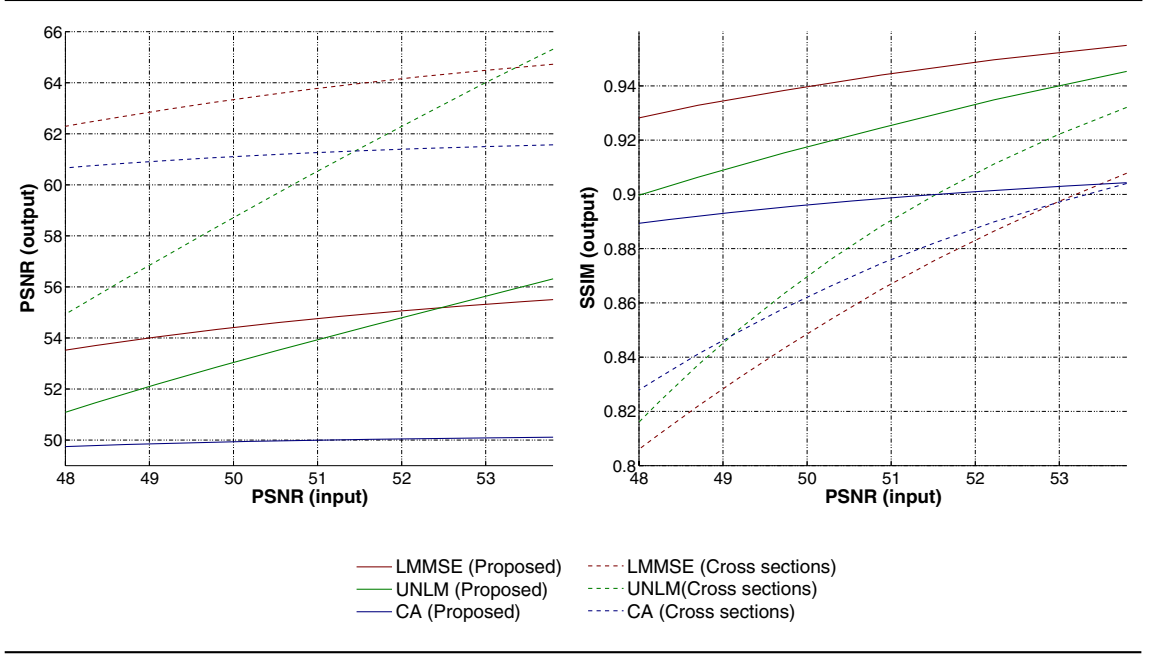

Fig. 3. Filtering performance (PSNR and SSIM) for Linear Minimum Mean Squared Error (LMMSE), Unbiased Non-Local Means (UNLM) and Conventional Approach (CA) filters, for a simple cross sections phantom and the proposed phantom. Results are shown for 15 gradient directions.

synthetic gradient images $(\mathbf{d})$ are cleaner and have a realistic contrast. On the contrary, the baseline image obtained from UNLM filtering and averaging (e) has an excellent quality, since the original baselines are far less noisy than the original gradient images. Although the synthetic gradients are very clean, some outliers may be appreciated in $\mathbf{d}$, so median filtering is used to obtain the final phantom f. Nevertheless, note that the majority of outliers are located in the background and the CSF (where no fiber bundles are present), which should be removed for the assessment of filtering performance. The final result (e and $\mathbf{f}$ ) is a very clean image, but yet it has well defined borders, being an appropriate phantom. In $\mathbf{g}$ and $\mathbf{h}$ we show noise corrupted images, in a high $\mathrm{SNR}^{1}$ ( $\mathbf{g}$ ) and a low SNR case $(\mathbf{h})$. Note that practically any realistic situation will lie between these two extreme cases. For the correlation filter, we use $\eta^{2}=0.8^{2}$, estimated from the original image (a), so the noisy spots accurately resemble those in a.

To show the importance of an adequate phantom, we have intended an illustrative example. We are going to numerically compare different filtering algorithms for DWI, using the phantom here presented and a more typical phantom based on cross sections; the phantom is a clone of the 3D cross described in [17], but we have used 15 gradient directions and scaled the magnitude of the baseline and the gradient images so that they all show mean values equal to the mean values of our own phantom. Regarding the algorithms, we have chosen two recent approaches: the popular UNLM implemented as described in [15], with the

${ }^{1}$ We use the Peak Signal to Noise Ratio (PSNR) (maximum SNR in the baseline image) to give numeric results in all cases. 
parameters proposed in Section 3.2, and the LMMSE filter described in [17] with the code issued by the authors and the parameters suggested in [17]. Additionally, we test the Conventional Approach (CA), see [9], implemented as an isotropic Gaussian low-pass filter with $\eta=1.5$ applied to the squared signal, followed by bias removal and computation of the squared root. As similarity measures, we use the PSNR and the Structural Similarity (SSIM) index 20] between the filtered volume and the ground truth (see Fig. 2-f). Results may be found in Fig. 3. CA, although being a very simple approach, shows better results than UNLM for PSNR (for low input SNR) and than LMMSE for SSIM (practically for all input SNR) when using the cross sections phantom. When we use our realistic phantom, CA obviously shows worse results than the other filters, since it is not designed to preserve the borders. In the cross sections phantom there are practically no borders, so this is not an issue. If we center our attention in the SSIM index, it is worth notice that the two phantoms give completely opposite results for UNLM and LMMSE for all input PSNR; the realistic phantom shows that LMMSE shows a better behavior than UNLM for this particular index, but using the over-simplified phantom would lead to the erroneous conclusion that UNLM yields better SSIM indices. Although this behavior cannot be generalized from this single experiment, it is useful to show the bias that an unrealistic phantom may induce.

\section{Conclusions}

A methodology to build a realistic DWI phantom to assess the accuracy of filtering algorithms for DWI has been described. Contrary to other validation methods proposed in the literature, see [14, our methodology does not require multiple observations of the same volume; the phantom may be built from one single DWI data-set. Moreover, the same real-data volume may be used to produce phantoms with very different configurations (different $b$ values and numbers of gradient directions) as described in Section 3.4. so it may be used to test filtering algorithms in a wide variety of situations. On the other hand, we have shown an example of how an inadequate phantom may lead to erroneous conclusions in the assessment of filtering performance, which justifies the importance of the work presented.

Acknowledgments. Authors would like to acknowledge grant number TEC2007 -67073/TCM from the Comisión Interministerial de Ciencia y Tecnología (Spain).

\section{References}

1. Stejskal, E.-O., Tanner, J.-E.: Spin diffusion measurements: Spin echoes in the presence of a time-dependent field gradient. J. of Chem. Phys. 42, 288-292 (1965)

2. Basser, P.J., Pierpaoli, C.: Microstructural and physiological features of tissues elucidated by Quantitative-Diffusion-Tensor MRI. J. of Magn. Res. 111(3), 209219 (1996) 
3. Descoteaux, M., Angelino, E., Fitzgibbons, S., Deriche, R.: Regularized, fast, and robust analytical Q-Ball imaging. Magn. Res. in Med. 58, 497-510 (2007)

4. Gudbjartsson, H., Patz, S.: The Rician distribution of noisy MRI data. Magn. Res. in Med. 34, 910-914 (1995)

5. Basser, P.J., Pajevic, S.: Statistical artifacts in diffusion tensor MRI (DT-MRI) caused by background noise. Magn. Res. in Med. 44, 41-50 (2000)

6. Jones, D., Basser, P.J.: Squashing peanuts and smashing pumpkins: how noise distorts diffusion-weighted MR data. Magn. Res. in. Med. 52, 979-993 (2004)

7. Pennec, X., Fillard, P., Ayache, N.: A Riemannian framework for tensor computing. Intl. J. of Com. Vision 66, 41-66 (2006)

8. Fillard, P., Pennec, X., Arsigny, V., Ayache, N.: Clinical DT-MRI estimation smoothing, and fiber tracking with log-Euclidean metrics. IEEE Trans. on Med. Imag. 26, 1472-1482 (2007)

9. McGibney, G., Smith, M.: Unbiased signal to noise ratio measure for magentic resonance images. Med. Phys. 20(4), 1077-1078 (1993)

10. Sijbers, J., den Dekker, A.-J.: Maximum Likelihood estimation of signal amplitude and noise variance from MR data. Magn. Res. Imag. 51, 586-594 (2004)

11. Parker, G.J., Schnabel, J.A., Symms, M.R., Werring, D.J., Barker, G.J.: Nonlinear smoothing for reduction of systematic and random errors in diffusion tensor imaging. J. of Magn. Res. Imag. 11, 702-710 (2000)

12. Pižurica, A., Philips, W., Lemahieu, I., Acheroy, M.: A versatile Wavelet domain noise filtration technique for medical imaging. IEEE Trans. on Med. Imag. 22(3), 323-331 (2003)

13. McGraw, T., Vemuri, B.C., Chen, Y., Mareci, T., Rao, M.: DT-MRI denoising and neural fiber tracking. Med. Imag. Anal. 8, 95-111 (2004)

14. Wiest-Daesslé, N., Prima, S., Coupé, P., Morrissey, S.P., Barillot, C.: Non-local means variants for denoising of diffusion-weighted and diffusion tensor MRI. In: Ayache, N., Ourselin, S., Maeder, A. (eds.) MICCAI 2007, Part II. LNCS, vol. 4792, pp. 344-351. Springer, Heidelberg (2007)

15. Wiest-Daesslé, N., Prima, S., Coupé, P., Morrissey, S.P., Barillot, C.: Rician noise removal by Non-Local Means filtering for low signal-to-noise ratio MRI: Applications to DT-MRI. In: Metaxas, D., Axel, L., Fichtinger, G., Székely, G. (eds.) MICCAI 2008, Part II. LNCS, vol. 5242, pp. 171-179. Springer, Heidelberg (2008)

16. Aja-Fernández, S., Niethammer, M., Kubicki, M., Shenton, M.E., Westin, C.-F.: Restoration of DWI data using a Rician LMMSE estimator. IEEE Trans. on Med. Imag. 27(10), 1389-1403 (2008)

17. Tristán-Vega, A., Aja-Fernández, S.: Joint LMMSE estimation of DWI data for DTI processing. In: Metaxas, D., Axel, L., Fichtinger, G., Székely, G. (eds.) MICCAI 2008, Part II. LNCS, vol. 5242, pp. 27-34. Springer, Heidelberg (2008)

18. Martín-Fernández, M., Muñoz-Moreno, E., Cammoun, L., Thiran, J.-P., Westin, C.-F., Alberola-López, C.: Sequential anisotropic multichannel Wiener filtering with Rician bias correction applied to 3D regularization of DWI data. Med. Imag. Anal. 13, 19-35 (2009)

19. Collins, D.L., Zijdenbos, A.P., Kollokian, V., Sled, J.G., Kabani, N.J., Holmes, C.J., Evans, A.C.: Design and construction of a realistic digital brain phantom. IEEE Trans. on Med. Imag. 17(3), 463-468 (1998)

20. Wang, Z., Bovik, A.-C., Sheikh, H.-R., Simoncelli, E.-P.: Image quality assessment: form error visibility to structural similarity. IEEE Trans. on Im. Process. 13(4), 600-612 (2004) 